\title{
Volumetric intensity modulated arc therapy for stereotactic body radiosurgery in oligometastatic breast and gynecological cancers: Feasibility and clinical results
}

\author{
GABRIELLA MACCHIA ${ }^{1}$, FRANCESCO DEODATO ${ }^{1}$, SAVINO CILLA ${ }^{2}$, GABRIELLA TORRE $^{3}$, \\ GIACOMO CORRADO ${ }^{4}$, FRANCESCO LEGGE ${ }^{4}$, MARIA ANTONIETTA GAMBACORTA ${ }^{5}$, \\ LUCA TAGLIAFERRI ${ }^{5}$, SAMANTHA MIGNOGNA ${ }^{3}$, GIOVANNI SCAMBIA ${ }^{6}$, \\ VINCENZO VALENTINI ${ }^{5}$, ALESSIO G. MORGANTI ${ }^{1,3,5^{*}}$ and GABRIELLA FERRANDINA ${ }^{4,6^{*}}$ \\ ${ }^{1}$ Radiotherapy, ${ }^{2}$ Medical Physics, ${ }^{3}$ Palliative Therapy and ${ }^{4}$ Gynaecologic Oncology Units, \\ Fondazione di Ricerca e Cura 'Giovanni Paolo II', Università Cattolica del Sacro Cuore, Campobasso; \\ Departments of ${ }^{5}$ Radiation and ${ }^{6}$ Gynaecologic Oncology, Università Cattolica del Sacro Cuore, Rome, Italy
}

Received April 2, 2014; Accepted July 4, 2014

DOI: 10.3892/or.2014.3412

\begin{abstract}
In the present study, the preliminary results of the first stereotactic body radiosurgery (SRS) experience with volumetric intensity modulated arc therapy (VMAT) in oligometastatic breast and recurrent gynecological tumors (OBRGT) are reported in terms of feasibility, toxicity and efficacy. Patients were treated in a head-first supine treatment position on a customized body frame immobilization shell. SRS-VMAT treatment plans were optimized using the ERGO++ treatment planning system. Response assessment was performed 8-12 weeks after treatment by morphologic imaging modalities, or if feasible, also by functional imaging. Thirty-six lesions in 24 consecutive patients (median age, 63 years; range, $40-81$ ) were treated: $13.9 \%$ had primary or metastatic lung lesions, $30.5 \%$ had liver metastases, $36.1 \%$ had bone lesions, $16.7 \%$ had lymph node metastases and $2.8 \%$ had a primary vulvar melanoma. The median dose was $18 \mathrm{~Gy}\left(\mathrm{BED}_{2 \mathrm{~Gy}, \alpha / \beta}: 10=50.4 \mathrm{~Gy}\right)$, the minimal dose was $12 \mathrm{~Gy}\left(\mathrm{BED}_{2 \mathrm{~Gy}, \alpha / \beta}: 10=26.4 \mathrm{~Gy}\right)$ and the maximal dose was 28 Gy $\left(\mathrm{BED}_{2 \mathrm{~Gy}, \alpha / \beta}: 10=106.4 \mathrm{~Gy}\right)$. Seven patients $(29.2 \%)$ experienced acute toxicity, which however was grade 2 in only 1 case. Moreover, only 3 patients (12.5\%) developed late toxicity of which only 1 was grade 2 . Objective response rate
\end{abstract}

Correspondence to: Dr Francesco Deodato, Radiation Oncology Unit, Fondazione di Ricerca e Cura 'Giovanni Paolo II', Università Cattolica del Sacro Cuore, Campobasso, Largo A. Gemelli 1, I-86100 Campobasso, Italy

E-mail: fdeodato@rm.unicatt.it

${ }^{*}$ Contributed equally

Key words: stereotactic body radiosurgery, recurrent gynaecologic tumors, volumetric intensity modulated arc therapy, oligometastatic breast cancer was 77.7\% including 16 lesions achieving complete response (44.4\%) and 12 lesions achieving partial response (33.3\%). The median duration of follow-up was 15.5 months (range, 6-50). Recurrence/progression within the SRS-VMAT treated field was observed in 6 patients (total lesions $=7$ ) with a 2-year inside SRS-VMAT field disease control expressed on a per lesion basis of $69 \%$. Recurrence/progression of disease outside the SRS-VMAT field was documented in 15 patients; the 2-year outside SRS-VMAT field metastasis-free survival, expressed on a per patient basis, was $35 \%$. Death due to disease was documented in 6 patients and the 2-year overall survival was $58 \%$. Although the maximum tolerated dose was not reached, SRS-VMAT resulted in positive early clinical results in terms of tumor response, local control rate and toxicity.

\section{Introduction}

Oligometastatic breast or recurrent gynecological tumor (OBRGT) patients, if inoperable, have a poor prognosis particularly when multiple therapeutic approaches have been already attempted and further oncologic treatment may contribute to unacceptable morbidity.

In the last few years, locoregional radiation treatment of oligometastatic disease in gynecological and breast cancer has become more prevalent (1-4).

In this context, radiotherapy should be ideally able to deliver the entire dose necessary for the ablative intent in the shortest delivery time. To date, this goal may be obtained by combining two fundamental technological advances: stereotactic body radiotherapy (SBRT), known as stereotactic body radiosurgery (SRS) when administered in a single fraction $(5,6)$ and intensity modulated radiotherapy with volumetric arc technique known with the acronym of VMAT.

Briefly, SBRT couples a high degree of anatomic targeting accuracy and reproducibility with very high doses of precisely delivered radiation, thereby maximizing the cell killing effect on the target(s) while minimizing radiation-related injury in adjacent normal tissues (7). Few experiences have been 
reported concerning the use of stereotactic techniques for administering an ablative dose in 3-5 fractions in recurrent gynecological tumors (1-3,8-13). Nevertheless, the available data allow consideration of this technique as a promising palliative treatment strategy for OBRGT due to the achievement of a $>80 \%$ local control (LC) rate and a low incidence of serious toxicity despite the high dose fractions of administered radiation $(1-3,10,12,13)$.

Moreover, VMAT, a novel technique characterized by dynamic arc dose delivery (14), administers radiation through a rotational movement of the linear accelerator gantry, while a continuous variation in beam profile and intensity is obtained. The expected advantages of this approach are represented by increased delivery efficiency and reduced risk of intra-fraction deviations both in terms of set-up errors and organ motion.

Therefore, VMAT may represent a valuable technique for SRS treatment; however, while little evidence exists concerning the feasibility of SRS-VMAT in different clinical settings (15), no data concerning SRS-VMAT have been reported in the treatment of OBRGT.

In the context of our continuing efforts to improve treatment efficacy in oligometastatic cancer patients $(3,16)$, we launched a prospective phase I clinical trial (DESTROY-2), aimed at primarily defining the maximum tolerated dose (MTD) of SRS-VMAT in patients with oligometastatic disease; secondary objectives are represented by feasibility evaluation in terms of dose-volume constraints, analysis of correlation between dosimetric and toxicity data, clinical response and assessment of local control rate (15).

Herein, we present the preliminary results of feasibility, toxicity and efficacy of our SRS-VMAT experience in OBRGT patients.

\section{Materials and methods}

This is a preliminary analysis of feasibility, toxicity and clinical efficacy of SRS-VMAT administered to a cohort of OBRGT patients enrolled in the DESTROY-2 study, as previously reported (15).

Eligibility. The DESTROY-2 trial was approved by the local Ethics Committee and the Institutional Review Board (P\#988/CE/2010) and all patients signed a written informed consent.

Patients who entered the present analysis were selected among patients enrolled from August 2010 and March 2013 into the DESTROY-2 protocol, and inclusion criteria were: age $>18$ years, diagnosis of breast and gynecologic cancer recurrences not indicated for resection or other locally ablative treatments, ECOG performance status $\leq 3$, adequate bone marrow function (neutrophil $>1,500 / \mathrm{mm}^{3}$, platelets $>100,000 / \mathrm{mm}^{3}$ ), adequate renal function (blood urea nitrogen $<25 \mathrm{mg} / \mathrm{dl}$, creatinine $<1.5 \mathrm{mg} / \mathrm{dl}$ ), normal liver function (bilirubin $<3 \mathrm{mg} / \mathrm{dl})$.

Exclusion criteria were as follows: $\mathrm{ECOG}>3$, uncontrolled severe infection and/or medical problems unrelated to malignancy, severe heart disease (if thorax site), diverticulitis or ulcerative recto-colitis or pelvic inflammatory diseases (if pelvic site). Patient should not have received SRS-VMAT prior to clinical trial enrollment.
Simulation. Treatment set-up was performed with a CT simulator. Women were treated in a head-first supine treatment position with arms at their sides while lying on a customized body-frame immobilization shell (Elekta Stereotactic BodyFrame or SBF; Elekta Oncology Systems, Crawley, UK). To evaluate the reproducibility of the set-up, three CT scan evaluations were performed on three different days, aimed to verify set-up deviation $<3 \mathrm{~mm}$. Moreover, to evaluate the organ motion, target displacement was measured performing 30 free breathing axial CT scans on the same slice. For displacement $>5 \mathrm{~mm}$, the SBF abdominal compressor was applied and the CT scan for organ motion assessment was repeated. The final CT simulation, for the acquisition of axial images necessary for stereotactic localization and plan calculations, was produced with a spiral technique. Three-millimeter scans were acquired with a $3-\mathrm{mm}$ interval between scans in the target region. For treating abdominal or pelvic targets, patients received $2 \mathrm{cc}$ of oral Gastrografin, diluted in $500 \mathrm{cc}$ water $30 \mathrm{~min}$ before CT scan. In the case of mediastinal, abdominal or pelvic target volumes, intravenous infusion of an iodinated contrast medium was also used.

Target and normal tissue contouring. The contoured radiosurgical gross tumour volume (GTV) consisted of identified cancer target(s), highlighted by $\mathrm{CT}$ and/or ${ }^{18} \mathrm{~F}$-FDG PET and/or MRI and agreed upon by both the treating radiation oncologist and a gynecologic oncologist. Each planning target volume (PTV) was individually defined for each patient as follow: the internal margin was based on respiratory excursions and the set-up margin was set at $3 \mathrm{~mm}$ according to the ROSEL study (17). Nearby normal tissue structures (OARs) according to the irradiated site were contoured by the radiation oncologist or a certified medical dosimetrist.

Treatment planning and dose delivery. SRS-VMAT treatment plans were optimized using the ERGO++ treatment planning system with VMAT technique (Elekta). All plans were generated with a single arc clockwise rotation, described in the optimization process by a sequence of 86 control points, i.e. one every $4^{\circ}$. The dose calculation was performed using the pencil beam algorithm with inhomogeneity correction and a dose grid resolution of $2 \mathrm{~mm}$. A uniform method for the selection of the prescription isodose surface (IDS) was used. According to the ROSEL study (17), for each plan, the IDS was selected as the greatest IDS fulfilling the two following criteria: $95 \%$ of the PTV volume reached $100 \%$ of the prescription dose and $99 \%$ of the PTV reached $\geq 90 \%$ of the prescription dose. The maximum dose within the PTV should not exceed $140 \%$ of the prescribed dose. Careful attention was paid to ensure that the maximum dose always remained within the GTV according to OAR constraints, previously reported in detail (15). SRS-VMAT plans were exported to the record and verify $(\mathrm{R} \& \mathrm{~V})$ system, Mosaiq v. 1.6 (Impac Software; Elekta) by DICOM-RT for later irradiation. Radiation prescription doses ranged from 12 to $28 \mathrm{~Gy}$ according to different arms of the dose escalation protocol as detailed elsewhere (15). All plans underwent dosimetric verification by means of ion chamber array, using gamma-analyses (18). For all patients, portal images before irradiation were acquired on virtual orthogonal beams. Deviations $>3 \mathrm{~mm}$ in the isocenter position 
were immediately corrected. For quality assurance through treatment planning and delivery, two independent checks (IC1 and 2) were performed by the medical and physics staff, as previously described (19).

Supportive therapy. Patients receiving thorax irradiation were given prescriptions for $0.5 \mathrm{mg}$ oral betamethasone, 3 times/ day for 1 month, followed by a gradual reduction, which are associated with gastric protection (H2-inhibitors). Patients receiving abdominal irradiation were prescribed $10 \mathrm{mg}$ oral metoclopramide, 3 times/day, for $\leq 1$ week following radiation therapy and $40 \mathrm{mg}$ oral rabeprazole, daily for 12 months (in case of stomach and/or duodenum irradiation). In addition, patients with upper abdomen lesions received $3 \mathrm{mg}$ intravenous granisetron plus $12 \mathrm{mg}$ intravenous dexamethasone immediately before radiosurgery and $6 \mathrm{~h}$ later.

Evaluation of response and follow-up. Tumor response assessment was performed 8-12 weeks after treatment. Morphologic imaging modalities were employed (CT with contrast medium and/or MRI with or without contrast) in all patients. Tumour response was based on the Response Evaluation Criteria in Solid Tumours (RECIST) criteria (20). If feasible, the response was also assessed by functional imaging, which included $\left({ }^{18} \mathrm{~F}\right)$-fluorodeoxyglucose (FDG)-PET. Herein, the European Organisation for Research and Treatment of Cancer (EORTC) criteria (21) were used as previously detailed (15).

Follow-up was performed 2 weeks after SRS-VMAT to evaluate acute toxicity, 3 months later to evaluate response by abdominal CT and PET-CT and every 6 months thereafter.

Toxicity and quality of life (QoL) evaluation. Adverse events were prospectively assessed. Grading of toxicity was based on the Common Terminology Criteria for Adverse Events (CTCAE V4.03), with the highest grade of any observed toxicity reported for each patient (22). Several parameters were recorded to evaluate the SRS-VMAT impact on pain, ECOG performance status, weight and QoL by nursing staff. QoL indices were evaluated using cancer linear analog scales, for well-being (CLAS1), fatigue (CLAS2) and ability to perform daily activities (CLAS3), respectively (23). The visual analog scale (VAS) for pain (24), the pain score (pain evaluation obtained by multiplying severity $\mathrm{x}$ frequency) and the drugs score (analgesic assumption evaluation obtained by multiplying severity $\mathrm{x}$ frequency) were used to record and monitor pain (25).

Statistical analysis. Objective response rate (ORR) included complete and partial response. Clinical benefit included ORR and stabilization of disease. The $95 \%$ confidence intervals (95\% CI) have been provided.

Local control (LC) of irradiated lesions was calculated using the Kaplan-Meier method (26) from the date of SRS-VMAT to the date of the inside SRS-VMAT field relapse/progression of disease or the date last seen. Metastasis-free survival (MFS) was calculated on a per patient basis from the date of SRS-VMAT to the date of relapse/progression of disease outside SRS-VMAT field or the date last seen. Overall survival (OS) was calculated on a per patient basis from the date of SRS-VMAT to the date of death or the date of the last visit.
Statistical analysis was carried out using SOLO (BMDP Statistical Software, Los Angeles, CA, USA).

\section{Results}

Patient characteristics. Twenty-four patients meeting the inclusion criteria and irradiated between August 2010 and March 2013 were analyzed. Clinical and pathological characteristics of the patients are summarized in Table I. The median age was 63 years (range, 40-81) and the vast majority of patients $(\mathrm{N}=18,75.0 \%)$ had ECOG performance status $<2$. Patients were considered as overweight/obese in $29.2 \%$ of cases. The primary tumor was most frequently represented by breast carcinoma $(75.0 \%)$, followed by endometrial $(8.3 \%)$ and cervical cancer $(8.3 \%)$. Up to two-thirds of patients suffer from comorbidities such as hypertension, diabetes or vasculopathies. All patients were studied for quality of life using CLAS score system, VAS and clinical parameters as pain score, drug score. Seven patients $(29.1 \%)$ had been previously irradiated at the SRS-VMAT site and $3(12.5 \%)$ had already received $>50$ Gy to the SRS-VMAT site. Table II documents the details concerning the SRS-VMAT sites, doses and treatment volume data. Doses were according to the DESTROY-2 dose escalation protocol, as previously detailed (15).

Fourteen patients received radiosurgery on a single lesion, while 8 and 2 patients were irradiated on 2 and 3 different metastatic sites, respectively, thus leading to a total number of 36 lesions including lung (13.9\%), liver (30.5\%), vulva (2.8\%), lymph node $(16.7 \%)$ and bone $(36.1 \%)$ (Table II).

Median dose was $18 \mathrm{~Gy}\left(\mathrm{BED}_{2 \mathrm{~Gy}, \alpha / \beta: 10}=50.4 \mathrm{~Gy}\right)$, minimal dose was $12 \mathrm{~Gy}\left(\mathrm{BED}_{2 \mathrm{~Gy}, \alpha / \beta: 10}=26.4 \mathrm{~Gy}\right)$ and maximal dose was 28 Gy $\left(\mathrm{BED}_{2 \mathrm{~Gy}, \alpha / \beta} \mathrm{B}_{10}=106.4 \mathrm{~Gy}\right)$. Median SRS-VMAT GTV was $4.4 \mathrm{cc}$ (range, 0.1-42.3 cc), while the median SRS-VMAT PTV ranged from 3.7 to $133.4 \mathrm{cc}$ (median, $19.4 \mathrm{cc}$ ). The dose/volume constraints (15) were respected in all lesions.

Safety. All patients received prescribed SRS-VMAT treatment and were included in the safety analysis: 7 patients $(29.2 \%)$ experienced grade 1-2 acute toxicity, which however was grade 2 in only 1 case. Moreover, only 3 patients $(12.5 \%)$ developed grade 1-2 late toxicity of which only 1 was grade 2 . Details concerning the time of onset, type and severity of complications are provided in Table III. Acute adverse events included asymptomatic pneumonitis not requiring intervention $(n=1)$, grade 1 skin toxicity $(n=2)$ and grade 2 mucositis $(n=1)$ causing discomfort, oedema and redness successfully treated with topic medications. Mild pain worsening in the irradiated site (flare-up) was reported in $3(12.5 \%)$ patients. Regarding late toxicity, we observed 1 case of grade 2 symptomatic pneumonitis out of 5 patients treated on the thorax, and this patient was treated by oral steroids. Neither grade 3-4 toxicities nor treatment-related deaths were reported.

Efficacy. All 36 irradiated lesions were evaluated for best response (Table IV). The ORR of target lesions to SRS-VMAT was $77.7 \%$ including 16 lesions achieving complete response (44.4\%) and 12 lesions achieving partial response $(33.3 \%)$. Stabilization of disease (SD) was observed in 7 lesions (19.4\%), while progression inside SRS-VMAT field was documented in 
Table I. Characteristics of the entire study population.

\begin{tabular}{|c|c|c|c|c|c|}
\hline Characteristics & $\begin{array}{l}\text { Patients } \\
\mathrm{n}(\%)\end{array}$ & $\begin{array}{c}\text { Target lesions } \\
\mathrm{n}(\%)\end{array}$ & Characteristics & $\begin{array}{c}\text { Patients } \\
\mathrm{n}(\%)\end{array}$ & $\begin{array}{c}\text { Target lesions } \\
\mathrm{n}(\%)\end{array}$ \\
\hline Total & $24(100)$ & $36(100)$ & CLAS 3 & & \\
\hline Age, years & & & $\leq 5$ & $3(12.5)$ & \\
\hline Median (range) & $63(40-81)$ & & $>5$ & $21(87.5)$ & \\
\hline ECOG PS status $^{a}$ & & & Previous radiotherapy & & \\
\hline 0 & $18(75.0)$ & & on SRS site & $7(29.2)$ & $8(22.2)$ \\
\hline 1 & $3(12.5)$ & & $\mathrm{RT} \geq 50$ Gy to SRS site & $3(12.5)$ & $3(8.3)$ \\
\hline
\end{tabular}

Body mass index, $\mathrm{kg} / \mathrm{m}^{2}$

$\begin{array}{ll}18.5-24.9 & 9 \\ 25.0-28.9 & 8 \\ 30-34.9 & 2 \\ 25.0-39.9 & 1 \\ \geq 40.0 & 4\end{array}$

Primary tumor

Breast cancer

Endometrial cancer

Cervical cancer

Ovarian cancer

Vulvar cancer

Histotype

Breast lobular carcinoma

Breast ductal carcinoma

Breast ductal-lobular

carcinoma

Endometrial clear cell

carcinoma

Squamous cervical

carcinoma

Cervical adenocarcinoma

Serous ovarian carcinoma

Vulvar melanoma

8

2

1

4

$18(75.0)$

$2(8.3)$

$2(8.3)$

1 (4.2)

1 (4.2)

$3(12.5)$

$13(54.1)$

$2(8.3)$

2 (8.3)

27 (75.0)

$4(11.1)$

3 (8.3)

1 (2.7)

1 (2.7)

$6(16.7)$

$18(50.0)$

$3(8.4)$

4 (11.2)

1 (4.2)

2 (5.6)

1 (4.2)

1 (4.2)

1 (4.2)

Comorbidities

No

$8(33.3)$

$16(66.6)$

$17(70.8)$

$7(29.1)$

$\geq 1$

Drug score

0

$\geq 1$

$21(87.5)$

$3(12.5)$

$17(70.8)$

5 (20.8)

2 (8.3)

$\geq 5$

\section{CLAS 1}

$\leq 5$

$>5$

$8(33.3)$

$16(66.6)$

\section{CLAS 2}

Table I Continued.

${ }^{a}$ The Eastern Cooperative Group (ECOG) performance status (PS) reflects individual daily living activities on a scale of 0 (fully active with symptoms) to 5 (deceased). SRS, stereotactic body radiosurgery; VAS, visual analog scale; CLAS, cancer linear analog scale.

Table II. Details concerning the SRS-treated lesions ( $\mathrm{N}=36)$.

\begin{tabular}{lr}
\hline SRS-treated lesions & $\mathrm{n}(\%)$ \\
\hline Site & \\
Lung & $5(13.9)$ \\
Liver & $11(30.5)$ \\
Vulva & $1(2.8)$ \\
Lymph nodes & $6(16.7)$ \\
Bone & $13(36.1)$ \\
Region of SRS-treated lesion & \\
Thorax & $17(47.2)$ \\
Abdomen & $11(30.5)$ \\
Pelvis & $6(16.7)$ \\
Other & $2(5.6)$ \\
Dose (Gy) & \\
12 & $4(11.1)$ \\
16 & $8(22.2)$ \\
18 & $13(36.1)$ \\
20 & $4(11.1)$ \\
26 & $3(8.3)$ \\
28 & $4(11.1)$ \\
GTV volume (cc) & \\
Median (range) & \\
PTV volume (cc) & \\
Median (range) & \\
\hline
\end{tabular}

SRS, stereotactic body radiosurgery. GTV, gross tumour volume; PTV, planning target volume.

only 1 lesion $(2.8 \%)$. The rate of clinical benefit (ORR + SD) was $97.2 \%$.

As of October 2013, the median duration of follow-up was 15.5 months (range, 6-50). Recurrence/progression within the SRS-VMAT-treated field was observed in 6 patients (total lesions, 7). As shown in Fig. 1, the 2-year 
Table III. Type of acute and late complications according to organ system and grade (CTC-AE v.4.0 scale).

\begin{tabular}{lll}
\hline $\begin{array}{l}\text { Organ system } \\
\text { toxicity }\end{array}$ & $\mathrm{n}$ & \\
\hline $\begin{array}{l}\text { Acute toxicity }(\mathrm{N}=7) \\
\quad\end{array}$ & & \\
Lung & 1 & Type \\
G1 & 2 & \\
Skin & 2 & Faint erythema or dry desquamation \\
G1 & 1 & \\
Mucosal tissue & 1 & Vaginal inflammation with mild discomfort, edema and redness \\
G2 & 3 & \\
Other & 3 & Flare-up: mild worsening of the pain in the irradiated site \\
G1 & & \\
Late toxicity $(\mathrm{N}=3)$ & & \\
Lung & 2 & \\
G1 & 1 & Diagnostic observation of asymptomatic pneumonitis: intervention not indicated \\
G2 & 1 & Symptomatic pneumonitis: medical intervention indicated; limited instrumental activities of daily living \\
Liver & 1 & \\
G1 & 1 & Diagnostic observation of hepatobiliary disorders: intervention not indicated \\
\hline
\end{tabular}

Table IV. Response to SRS-VMAT on a per lesion basis.

\begin{tabular}{lcc}
\hline Clinical response & $\begin{array}{c}\text { Target lesions } \\
\mathrm{n}(\%)\end{array}$ & $95 \% \mathrm{CI}$ \\
\hline Complete response & $16(44.4)$ & $28.2-60.6$ \\
Partial response & $12(33.3)$ & $17.9-48.7$ \\
Objective response & $28(77.7)$ & $63.7-91.7$ \\
Stable disease & $7(19.4)$ & $6.5-32.3$ \\
Clinical benefit & $35(97.2)$ & $91.8-102.6$ \\
Progressive disease & $1(2.8)$ & $-2.6-8.2$ \\
\hline
\end{tabular}

VMAT, volumetric intensity modulated arc therapy; SRS, stereotactic body radiosurgery; $\mathrm{CI}$, confidence interval.

inside SRS-VMAT field disease control expressed on a per lesion basis, was $69 \%$.

Recurrence/progression of disease outside the SRS-VMAT field was documented in 15 patients; the 2-year outside SRS-VMAT field progression-free survival (PFS), expressed on a per patient basis, was $35 \%$. Death due to disease was documented in 6 patients and the 2-year OS was $58 \%$.

\section{Discussion}

To the best of our knowledge, this series represents the first report on the toxicity and activity of SRS-VMAT in breast and gynecologic cancer recurrences not indicated for resection or other locally ablative treatments. Despite sample heterogeneity in terms of doses and histotypes, we found that the single fraction irradiation approach was feasible and tolerable with encouraging, although preliminary, clinical results. In particular, despite the ablative doses delivered
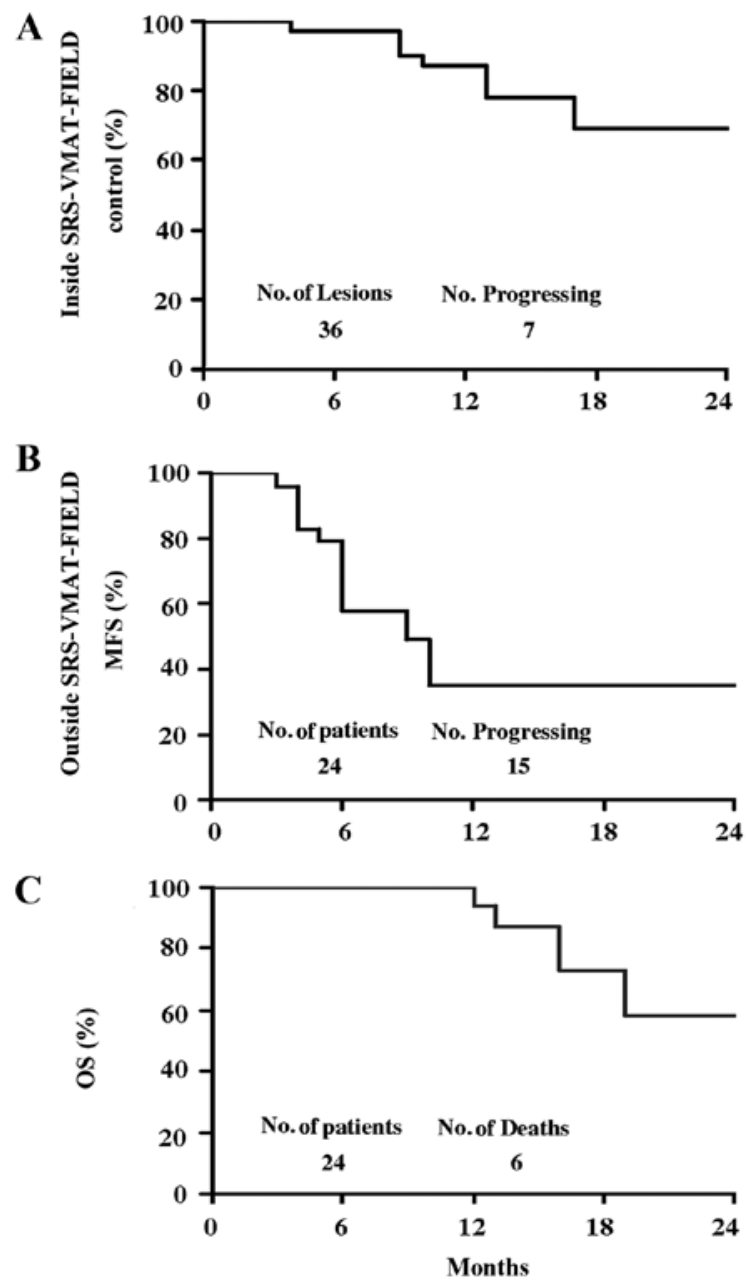

Figure 1. (A) The 2-year inside SRS-VMAT field disease control, expressed on a per lesion basis. (B) The 2-year outside SRS-VMAT field MFS, expressed on a per patient basis. (C) The 2-year OS, expressed on a per patient basis. SRS, stereotactic body radiosurgery; VMAT, volumetric intensity modulated arc therapy; MFS, metastasis-free survival; OS, overall survival. 
(median dose $=18 \mathrm{~Gy} ; \mathrm{BED}_{2}$ Gy, $\alpha \beta$ : $10=50.4 \mathrm{~Gy}$ ), only 1 case of acute grade 2 toxicity and 1 case of late grade 2 toxicity were observed; these results have to be taken into account considering that almost one-third of our cases had already been irradiated at the SRS-VMAT site. Retrospective studies employing SBRT for treatment of recurrent gynecological tumors have been reported in two literature reviews $(1,27)$; doses ranging between $14 \mathrm{~Gy}$ ( 2 fractions) and $45 \mathrm{~Gy}$ ( 3 fractions) were delivered with an acceptable safety profile with the exception of cases already submitted to previous treatments. In particular, Guckenberger et al (2), who delivered SBRT (15 Gy with 3 fractions by means of the IMRT technique) to oligometastatic gynecological patients already submitted to surgery and/or radiation, reported $>\mathrm{G} 2$ late toxicity in almost $25 \%$ of cases with 2 patients developing severe adverse events $(1$ grade 4 entero-vaginal fistula and 1 grade 4 small bowel ileus). Although the authors recognized that this rate of toxicity was similar to those reported with non-3D image guided brachytherapy \pm external beam radiotherapy (28-30), they also advocated the use of risk-adapted protocols, in which different SBRT fraction numbers with different single fraction doses are used depending on the proximity of targets and OARs, as for pulmonary SBRT in case of centrally located lung cancer (31).

Concerning breast cancer oligometastatic patients, systemic therapy or surgery, if feasible, are usually the main treatment for these patients. However, recently, Habermehl et $a l$ reported the safety of single-dose radiosurgical treatment for hepatic metastases, with 6 and 12 months local control of 87 and $70 \%$ (4). Considering the rapid increase in the scientific evidence in the relatively new field of stereotactic radiotherapy, we strongly believe that this technique in the next few years will be an integral part of the weapons available for the treatment of oligometastasized breast cancer.

In regards to the preliminary evaluation of treatment efficacy, the rate of ORR of the target lesions was $77.7 \%$ (complete response: $44.4 \%$ ); moreover, stabilization of tumor lesions was $\sim 97 \%$, respectively; these findings are in line with previously reported data obtained in similar settings with SBRT, which has provided response rates between 67 and $79 \%$ despite the use of various ablative doses and schedules $(1,3,4,9,10,13)$. Moreover, we reported a 2-year inside SRS-VMAT field local control rate of $69 \%$, when expressed on a per lesion basis.

Local control of SBRT-treated oligometastatic patients has been shown to range between 71 and $96 \%$ with 1-2 year follow up (32-35); explanations for this wide range of efficacy could be represented by the delivery of different doses and inclusion of various tumor types and histologies $(1,2,12,13)$. Moreover, the dose-finding design of our series as well as the choice to express the local control data on a per lesion basis must be taken into account when considering the comparison of our results with previous experiences.

As expected, the 2-year outside SRS-VMAT field-PFS and the 2-year OS were relatively dismal (35 and 58\%, respectively); as argued by Kunos et al (10) progression of disease elsewhere in the body shortly after SBRT may signal either progression of already present occult disease, or inability of SBRT to control targeted disease prior to disease dissemination, circumstances which both claim for concurrent chemotherapy administration. The low toxicity profile of SRS-VMAT technique and its fast administration makes this approach particularly suitable to be administered between one chemotherapy cycle and the other, probably resulting in a more effective, comprehensive approach to the oligometastatic setting. In this context, a phase I clinical trial of SBRT plus gemcitabine and carboplatin chemotherapy is underway (ClinicalTrials. gov identifier: NCT01652794). In addition to the higher acceptance and compliance to treatment of these vulnerable patients already faced with distressing experiences with several previous therapies, other potential advantages of SRS-VMAT must also be emphasized, such as lack of inter-fraction uncertainties that translate into a higher treatment reproducibility and reduced costs in spite of its complexity.

In conclusion, clinical radiation practice with SRS-VMAT for metastatic or previously irradiated gynecologic cancers appears promising and is likely to translate into a greater benefit for female cancer patient survival, although results from phase II trials will better assess tumor response.

\section{References}

1. Higginson DS, Morris DE, Jones EL, et al: Stereotactic body radiotherapy (SBRT): technological innovation and application in gynecologic oncology. Gynecol Oncol 120: 404-412, 2011.

2. Guckenberger M, Bachmann J, Wulf J, et al: Stereotactic body radiotherapy for local boost irradiation in unfavourable locally recurrent gynaecological cancer. Radiother Oncol 94: 53-59, 2010.

3. Deodato F, Macchia G, Grimaldi L, et al: Stereotactic radiotherapy in recurrent gynecological cancer: A case series. Oncol Rep 22: 415-419, 2009.

4. Habermehl D, Herfarth KK, Bermejo JL, et al: Single-dose radiosurgical treatment for hepatic metastases - therapeutic outcome of 138 treated lesions from a single institution. Radiat Oncol 8: 175, 2013.

5. Seung SK, Larson DA, Galvin JM, et al: American College of Radiology (ACR) and American Society for Radiation Oncology (ASTRO) practice guideline for the performance of stereotactic radiosurgery (SRS). Am J Clin Oncol 36: 310-315, 2013.

6. Solberg TD, Balter JM, Benedict SH, et al: Quality and safety considerations in stereotactic radiosurgery and stereotactic body radiation therapy: executive summary. Pract Radiat Oncol 2: 2-9, 2012.

7. Potters L, Kavanagh B, Galvin JM, et al: American Society for Therapeutic Radiology and Oncology (Astro) and American College of Radiology (ACR) practice guideline for the performance of stereotactic body radiation therapy. Int J Radiat Oncol Biol Phys 76: 326-332, 2010.

8. Molla M, Escude L, Nouet P, et al: Fractionated stereotactic radiotherapy boost for gynecologic tumors: an alternative to brachytherapy? Int J Radiat Oncol Biol Phys 62: 118-124, 2005.

9. Kunos C, Chen W, DeBernardo R, et al: Stereotactic body radiosurgery for pelvic relapse of gynecologic malignancies. Technol Cancer Res Treat 8: 393-400, 2009.

10. Kunos CA, Brindle J, Waggoner S, et al: Phase II clinical trial of robotic stereotactic body radiosurgery for metastatic gynecologic malignancies. Front Oncol 2: 181, 2012.

11. Kunos CA and Spelic M: Role of stereotactic radiosurgery in gynecologic cancer. Curr Opin Oncol 25: 532-538, 2013.

12. Jorcano S, Molla M, Escude L, et al: Hypofractionated extracranial stereotactic radiotherapy boost for gynecologic tumors: a promising alternative to high-dose rate brachytherapy. Technol Cancer Res Treat 9: 509-514, 2010.

13. Choi CW, Cho CK, Yoo SY, et al: Image-guided stereotactic body radiation therapy in patients with isolated para-aortic lymph node metastases from uterine cervical and corpus cancer. Int J Radiat Oncol Biol Phys 74: 147-153, 2009.

14. Otto K: Volumetric modulated arc therapy: IMRT in a single gantry arc. Med Phys 35: 310-317, 2008.

15. Deodato F, Cilla S, Macchia G, et al: Extracranial radiosurgery with volumetric modulated arc therapy: Feasibility evaluation of a phase I trial. Oncol Lett 5: 1889-1896, 2013. 
16. Macchia G, Morganti AG, Cilla S, et al: Quality of life and toxicity of stereotactic radiotherapy in pancreatic tumors: a case series. Cancer Invest 30: 149-155, 2012.

17. Hurkmans CW, Cuijpers JP, Lagerwaard FJ, et al: Recommendations for implementing stereotactic radiotherapy in peripheral stage IA non-small cell lung cancer: report from the quality assurance working party of the randomised phase III ROSEL study. Radiat Oncol 4: 1,2009.

18. Cilla S, Viola P, Azario L, et al: Comparison of measured and computer portal dose for IMRT treatment. J Appl Clin Med Phys 7: 65-79, 2006.

19. Morganti AG, Deodato F, Zizzari S, et al: Complexity index (COMIX) and not type of treatment predicts undetected errors in radiotherapy planning and delivery. Radiother Oncol 89: 320-329, 2008.

20. Therasse P, Arbuck SG, Eisenhauer EA, et al: New guidelines to evaluate the response to treatment in solid tumors. European Organization for Research and Treatment of Cancer, National Cancer Institute of the United States, National Cancer Institute of Canada. J Natl Cancer Inst 92: 205-216, 2000.

21. Young H, Baum R, Cremerius U, et al: Measurement of clinical and subclinical response using [18F]-fluorodeoxiglucose and positron emission tomography: review and 1999 EORTC recommendations. European Organisation for Research and Treatment of Cancer (EORTC) PET study group. Eur J Cancer 35: 1773-1782, 1999.

22. National Institutes of Health National Cancer Institute: Common Terminology Criteria for Adverse Events (CTCAE), version 4.03. Published on February 22, 2012.

23. Sutherland HJ, Walker P and Till JE: The development of a method for determining oncology patients' emotional distress using linear analogue scales. Cancer Nurs 11: 303-308, 1988.

24. Melzack R: The McGill pain questionnaire: major properties and scoring methods. Pain 1: 277-299, 1975.

25. Salazar OM, Sandhu T, da Motta NW, et al: Fractionated half-body irradiation (HBI) for the rapid palliation of widespread, symptomatic, metastatic bone disease: a randomized phase III trial of the International Atomic Energy Agency (IAEA). Int J Radiat Oncol Biol Phys 50: 765-775, 2001.
26. Kaplan EL and Meier P: Non-parametric estimation from incomplete observations. J Am Statist Assoc 53: 457-481, 1985.

27. Mayr NA, Huang Z, Sohn JW, et al: Emerging application of stereotactic body radiation therapy for gynecologic malignancies. Expert Rev Anticancer Ther 11: 1069-1075, 2011.

28. Grigsby PW: Radiotherapy for pelvic recurrence after radical hysterectomy for cervical cancer. Radiat Med 23: 327-330, 2005.

29. Haasbeek CJ, Uitterhoeve AL, van der Velden J, et al: Long-term results of salvage radiotherapy for the treatment of recurrent cervical carcinoma after prior surgery. Radiother Oncol 89: 197-204, 2008

30. Ito H, Shigematsu N, Kawada T, et al: Radiotherapy for centrally recurrent cervical cancer of the vaginal stump following hysterectomy. Gynecol Oncol 67: 154-161, 1997.

31. Guckenberger M, Wulf J, Mueller G, et al: Dose-response relationship for image-guided stereotactic body radiotherapy of pulmonary tumors: relevance of 4D dose calculation. Int J Radiat Oncol Biol Phys 74: 47-54, 2009.

32. Rusthoven KE, Kavanagh BD, Cardenes H, et al: Multi-institutional phase I/II trial of stereotactic body radiation therapy for liver metastases. J Clin Oncol 27: 1572-1578, 2009.

33. Rusthoven KE, Kavanagh BD, Burri SH, et al: Multi-institutional phase I/II trial of stereotactic body radiation therapy for lung metastases. J Clin Oncol 27: 1579-1584, 2009.

34. Mendez Romero A, Wunderink W, Hussain SM, et al: Stereotactic body radiation therapy for primary and metastatic liver tumors: a single institution phase i-ii study. Acta Oncol 45: 831-837, 2006.

35. Herfarth KK, Debus J, Lohr F, et al: Stereotactic single-dose radiation therapy of liver tumors: results of a phase I/II trial. J Clin Oncol 19: 164-170, 2001. 